\title{
Evaporative thermal resistance and its influence on microscopic bubble growth
}

\author{
Giovanni Giustini ${ }^{1}$, Satbyoul Jung ${ }^{2}$, Hyungdae $\mathrm{Kim}^{2}$, S P Walker ${ }^{1,3}$
}

\author{
${ }^{1}$ Department of Mechanical Engineering, Imperial College London, Exhibition Road, London \\ SW7 2AZ \\ ${ }^{2}$ Department of Nuclear Engineering, Kyung Hee University, Youngin, Republic of Korea \\ ${ }^{3}$ Corresponding author; s.p.walker@imperial.ac.uk
}

Simulations of the formation of small steam bubbles indicate that the rate of growth of bubbles is very sensitive to the rate of evaporation of the micro-layer of liquid beneath the bubble. Such evaporation is rapid, and is modelled as being driven by the large heat flux through the thin liquid layer caused by the difference in temperature between the solid-liquid interface, and the saturation temperature in the interior of the bubble. However, application of this approach to recent experimental measurements of Jung and Kim generated anomalous results. In this paper we demonstrate that a model of the micro-layer heat flux that includes an allowance for the finite evaporative thermal resistance is able to eliminate these anomalies. This evaporative thermal resistance is a consequence of near-interface molecular dynamics, characterised by a quantity termed 'evaporation coefficient'. Whilst in most engineering applications evaporative thermal resistance is small compared to conductive resistance, here, with the micro-layer thickness ranging from a few microns down to zero, it becomes of considerable importance. Selection of a molecular 'evaporation coefficient' to restore consistency to the anomalous measurements allows a plausible numerical value to be inferred. For the several times and multiple locations studied, a fairly consistent value of between 0.02 and 0.1 is indicated, (for saturated water in laboratory conditions), which itself is consistent with earlier literature values of this rather difficult quantity. It is shown that the evaporative resistance always represents a large fraction of the conductive resistance, and for important phases of the process dominates it. The need for inclusion of this phenomenon in the microlayer models used in bubble analysis is clear.

\section{INTRODUCTION}

\subsection{Our current understanding of the early stages of vapour bubble development}

Nucleate boiling has received considerable attention in recent years due to its intrinsic scientific interest, and the need for greater fundamental understanding [1]. From a practical point of view, also, a good predictive capability will be beneficial to inform macroscopic, component scale modelling of boiling in CFD [2].

In what is termed nucleate boiling, a vapour bubble nucleates at a solid wall from a preexisting pocket of gas or vapour (a nucleation site) and grows as steam is generated. Two asymptotic regimes of expansion are possible. Very early bubble growth is essentially an isothermal process [3], during which the factor limiting bubble growth rate is not the rate of production of vapour, but the rate at which momentum is transferred to the surrounding body of fluid ("inertial bubble growth"). Subsequent bubble growth is limited by the 
transport of heat to the bubble surface ("heat diffusion controlled bubble growth" [4]), and that is the focus of the present work.

When a vapour bubble grows on a superheated solid substrate steam is generated via two complementary mechanisms (Figure 1).

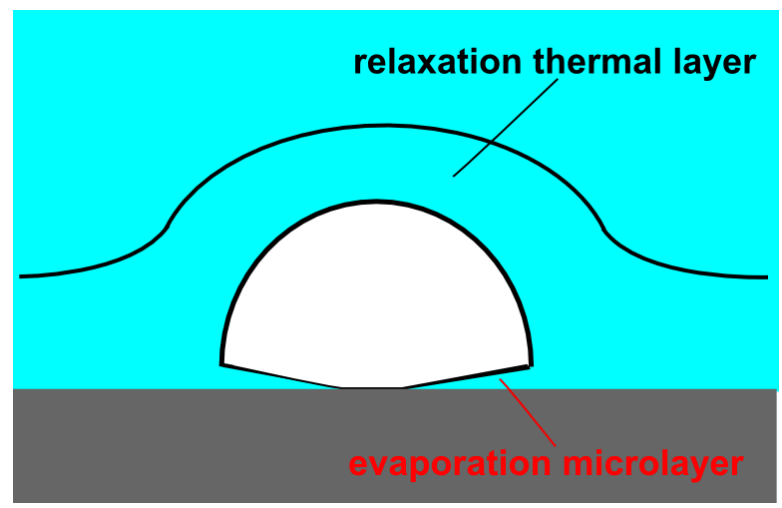

Figure 1

Current understanding of the early stages of bubble development, adapted from [5].

Much of the steam is generated by evaporation from the curved surface, via relaxation of the temperature of the superheated liquid layer surrounding the liquid-vapour interface.

As the bubble grows, (under at least some conditions) a thin layer of liquid is observed to be left beneath it [6] (Figure 2). This liquid film is generally termed the "micro-layer". Being such a thin layer, the flow of heat through it is large. This is because heat flow is driven by the temperature difference between the substrate-liquid interface and the liquid-vapour interface, and this difference exists over a very small distance [7]. Consequently, the micro-layer is believed to be responsible for a significant fraction of the vapour generation under many circumstances, such as the boiling of atmospheric water at a superheat around $10 \mathrm{~K}$ [8]. For water at atmospheric pressure, its radial extent is a few hundreds of microns, and its thickness a few microns, increasing slightly with radial distance from the nucleation site. It is believed that there is essentially no flow of liquid into or out of the micro-layer, and thus its evaporation results in its thickness reducing, and the eventual disappearance of the layer. Since this occurs first at its inner radius, where the initial thickness of the film was least, there is an associated progressive increase in the radius of the dry patch around the nucleation site [9]. 


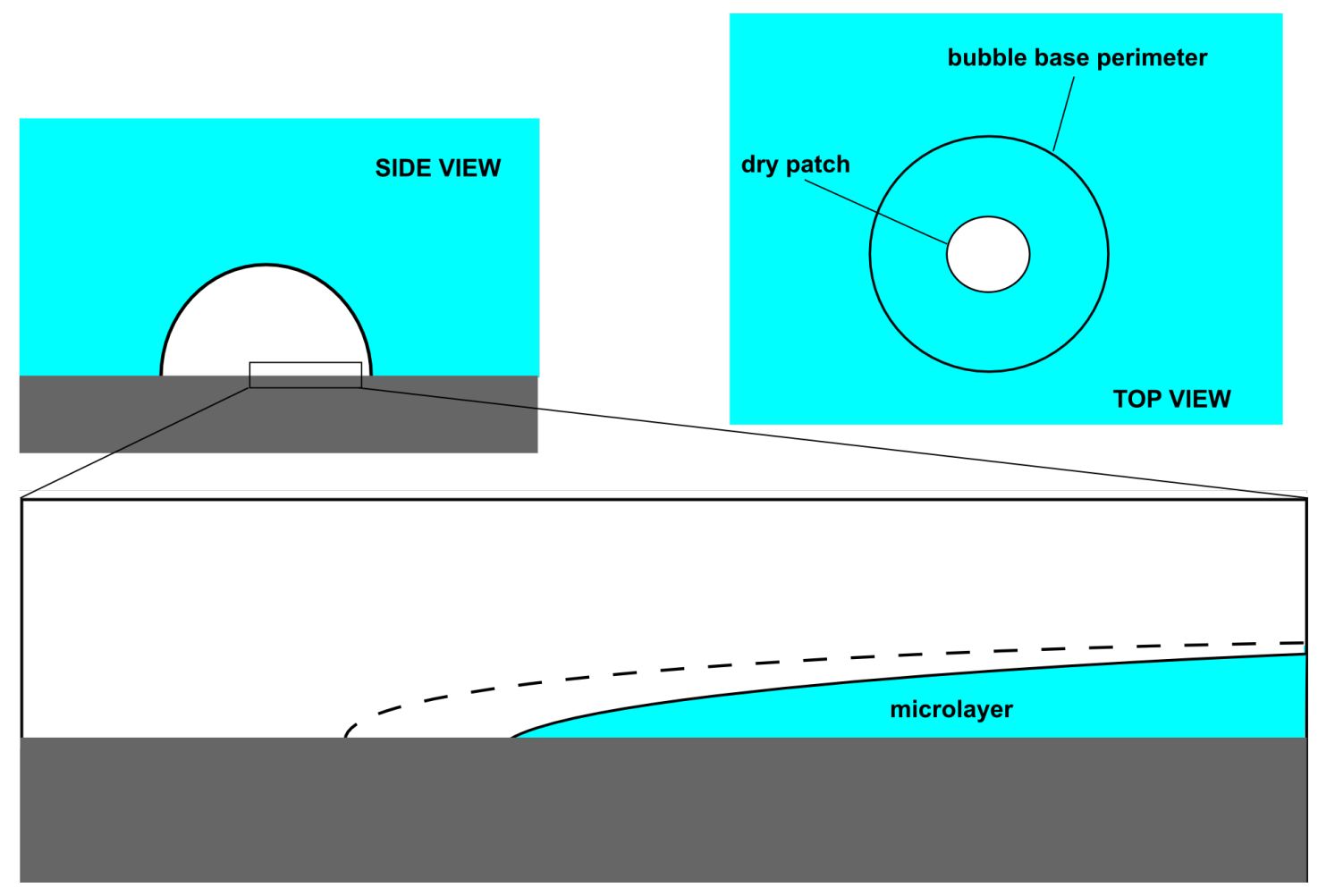

Figure 2

Current understanding of the micro-layer [10].

\subsection{Current modelling of the early stages of vapour bubble development}

The evaporation from the curved surface is limited by the rate at which heat diffuses across the relaxation layer. It is most common to take the liquid-vapour interface to be at the equilibrium saturation temperature at the system ambient pressure. (In a rather different approach [11], addressing largely refrigerants not water, some workers [12] have included deviations of the interface temperature from equilibrium, although micro-layer models did not feature in this analysis.)

The normal heat flux so computed in the liquid is used to determine a corresponding rate of vaporisation, and a corresponding vapour flux into the bubble [13].

The usual approach to modelling heat transfer through and vaporisation from the micro-layer is to treat it as a thin film, through which the heat flux is determined by its upper and lower surface temperatures, and where this heat flux in turn is used to compute the local vapour generation rate. This rate is used both to contribute to the increasing bubble volume, and to compute the gradual thinning and eventual disappearance of the micro-layer from the centre outwards.

\subsection{Evaporative thermal resistance}

In the above discussion, for both the main curved surface of the bubble and for the microlayer, the taking of the vapour-liquid interface temperature to be equal to the saturation temperature is equivalent to assuming that the evaporative heat transfer process is "infinitely effective", or equivalently can be modelled by an infinite value of heat transfer coefficient.

Some temperature difference is actually required to drive the flux of molecules from the liquid into the vapour. Expressing this in terms of an 'evaporative heat transfer coefficient', its value is indeed usually large, and the resistance to heat transfer it represents is a tiny 
fraction of the other resistances involved (such as the diffusive resistance impeding flow of heat from the bulk liquid to the bubble).

However, the thermal resistance of a thin micro-layer is itself small, and becomes vanishingly small as it thins. If the micro-layer plays a significant role in bubble development, neglect of the evaporative resistance might be expected to have a measurable influence on its contribution to predicted bubble growth.

In this work we will focus on the micro-layer during nucleate boiling of water at atmospheric pressure and investigate the significance of the inclusion of the evaporative thermal resistance.

In Section 2 we present a summary of the literature on the modelling of the evaporative thermal resistance. This is found to depend strongly on a free parameter termed an "evaporation" coefficient, albeit that this quantity is hard to measure, and consequently has highly uncertain values. In Section 3 we describe a well-established set of measurements of micro-layer evaporation and bubble growth. Analysis of these experiments, including the effect of evaporative thermal resistance, in presented in Section 4, where an estimate of the evaporation coefficient value is proposed based on the post-processing of experimental data. Conclusions are drawn in Section 5.

\section{PHYSICAL BASIS OF THE EVAPORATIVE RESISTANCE MODEL}

\subsection{Evaporation from a molecular point of view}

During both evaporation and condensation, the mass flux across the interface is driven by a temperature difference between the liquid surface and the vapour adjacent to the surface. For example, when the liquid side of the interface is hotter than the gas side, there is a net flux of molecules leaving the liquid and entering the gas because of this temperature difference. From a molecular point of view, this temperature difference, albeit presumably very small, is present even in the case of 'perfect' emission and absorption of molecules from the liquid surface. The term 'perfect' here denotes circumstances when all the molecules emitted from the liquid reach the vapour and stay there, and all the molecules impinging the liquid surface are absorbed. However, in general the emission or absorption of molecules from or into a liquid surface is not perfect, and scattering can take place. This is illustrated in Figure 3, adapted from [14].

Considering evaporation, the flux of molecules trying to escape the liquid surface depends on the liquid temperature. If scattering is present, such that some escaped molecules return, then to obtain the same flux of molecules reaching and remaining in the vapour as would depart the liquid in the absence of scattering a higher superheat of the liquid is required. In the next sections, we present a short survey on how this physical mechanism is incorporated in a simple model of the phase change process that can be used within the context of continuum mechanics. 


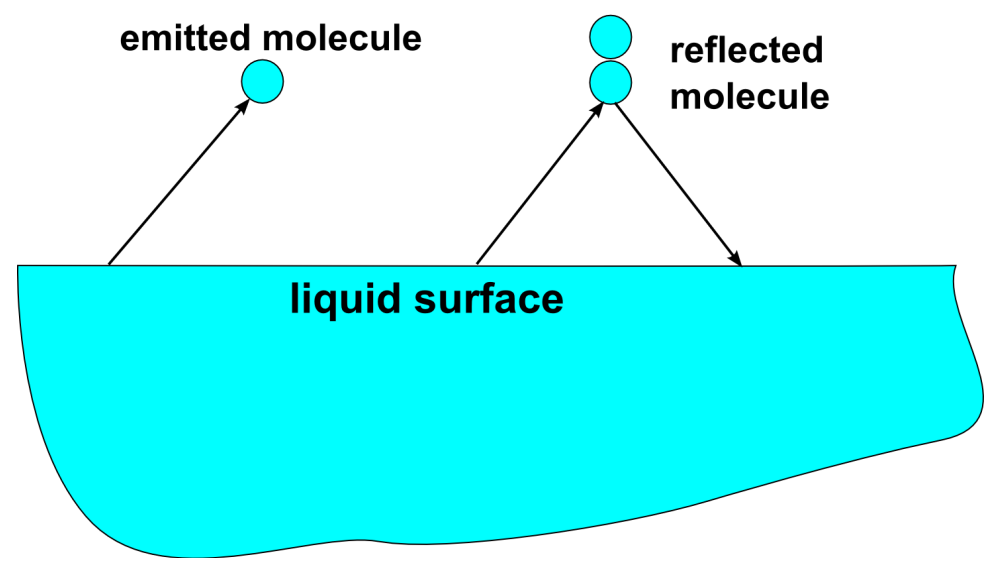

Figure 3

Evaporation from a molecular point of view.

\subsection{The representation of this as an effective heat transfer coefficient}

We follow here the development in Schrage [15]. Assuming that the vapour is an ideal gas and that the velocities of the molecules in the impinging and escaping fluxes follow a Maxwellian distribution, from the kinetic theory of gases one can obtain the following expression for the flux of molecules emitted from the liquid surface:

$$
\phi_{l}=\frac{p_{l}}{\sqrt{2 \pi R T_{l}}}
$$

or the flux of molecules from the vapour, impinging on the liquid surface:

$$
\phi_{v}=\frac{p_{v}}{\sqrt{2 \pi R T_{v}}}
$$

where $p_{l}$ and $p_{v}$ are the liquid and vapour pressures, and $T_{l}, T_{v}$ the liquid and vapour temperatures and $R$ is the gas constant.

The scattering process discussed above means that not all of this flux 'stays gone'; some molecules will return to their source region. For example, some molecules emitted from the liquid surface are caused to return to the liquid immediately by collision with extant vapour molecules. It is normal to introduce the quantities $f_{c}$, the fraction of molecules impinging on the liquid surface that are eventually absorbed, the 'condensation coefficient', and $f_{e}$, the fraction of molecules leaving the liquid surface that eventually reach the bulk vapour, the 'evaporation coefficient'.

There is an additional factor to consider including; an allowance for the bulk motion of molecules towards the liquid during condensation, or away from it during evaporation. From Tanasawa [16], for condensation this is given by:

$$
\Gamma=1+\frac{\dot{m}}{p_{v} \sqrt{2 /\left(\pi R T_{v}\right)}}
$$


where $\dot{m}$ is the net mass flux across the interface.

For atmospheric water at a condensation rate corresponding to a heat flux of $500 \mathrm{~kW} \mathrm{~m}^{-2}$, not untypical of micro-layer heat fluxes, the second term on the right hand side of (3) is approximately equal to 0.001 . Plainly for the cases of immediate interest it matters little, but it could perhaps become important under other circumstances.

Assuming this factor $\Gamma$ to be equal to one, for the case of evaporation the net flux of molecules from liquid to vapour is given by

$$
\dot{m}=f_{e} \phi_{l}-f_{c} \phi_{v}
$$

Schrage considered an interface at equilibrium (zero net mass flux) and concluded that $f_{e}=f_{c}$. He later applied the same finding to non-equilibrium conditions (finite net mass flux). We will adopt this approach here and also take $f_{e}=f_{c}=f$. Following Schrage, we term $f$ an 'evaporation coefficient'. Taking $p_{l}$ as the saturation pressure at the liquid surface temperature, the net mass flux can now be written as

$$
\dot{m}=f\left(\frac{p_{S A T}\left(T_{l}\right)}{\sqrt{2 \pi R T_{l}}}-\frac{p_{v}}{\sqrt{2 \pi R T_{v}}}\right)
$$

We will return to this expression later in the context of inferring a value for $f$ (section 4.2). Under the assumption that the temperature difference between the liquid surface and the vapour is small compared to their absolute values, one can use the Clausius-Clapeyron relation $\frac{p_{l}-p_{v}}{T_{l}-T_{v}}=\frac{\rho_{v} h_{f g}}{T_{v}}$ to obtain the following expression for the net mass flux across the interface as a function of fluid properties and interface superheat:

$$
\dot{m}=\frac{h_{f g} \rho_{v}\left(T_{l}-T_{v}\right)}{T_{v} \sqrt{2 \pi R T_{v}}} f
$$

The mass flux in this expression is readily converted into a heat flux. We will express the resulting relationship between interface superheat and heat flux via the evaporative thermal resistance

$$
T_{l}-T_{v}=\frac{T_{v} \sqrt{2 \pi R T_{v}}}{h_{f g}^{2} \rho_{v} f} \dot{q}^{\prime \prime}
$$

If the vapour is assumed to be at the saturation temperature at the system ambient pressure we then have

$$
T_{l}-T_{S A T}=\frac{T_{S A T} \sqrt{2 \pi R T_{S A T}}}{h_{f g}^{2} \rho_{v} f} \dot{q}^{\prime \prime}=\frac{1}{h_{e v}} \dot{q}^{\prime \prime}
$$

This provides an estimate for the evaporative heat transfer coefficient:

$$
h_{e v}=\frac{h_{f g}^{2} \rho_{v}}{T_{S A T} \sqrt{2 \pi R T_{S A T}}} f
$$


Its value is seen naturally to depend on the fluid properties, and in particular on the evaporation coefficient and the latent heat of evaporation.

\subsection{Typical evaporative heat transfer coefficient values}

Considerable difficulties are encountered when trying to measure the evaporation coefficient. This can be done only indirectly, for example by measuring the temperature of the liquid surface, and inferring the coefficient. Usually, favourable conditions to measure the evaporation coefficient are present when the gas phase is sufficiently rarified. For the case of water, this means that most of the measurements of the evaporation coefficient are at low pressures (tenths or hundredths of bars), and hence there is paucity of measurements of the evaporation coefficient for pressures equal to or above atmospheric. In addition, there is a large scatter of values suggested for the evaporation coefficient [14], due to the diverse experimental arrangements devised to infer values of it. In the analysis presented here, based on a survey of the literature [14-18], we will consider a large - but it seems fairly bounding range of evaporation coefficients, namely between 0.01 and 1 .

As discussed, the value of $f$ is rather uncertain, and we will now attempt to give a sense of the significance of this uncertainty. In Figure 4 we show the evaporative heat transfer coefficient $h_{e v}$ for water at atmospheric pressure, and, in terms relevant to the micro-layer, express this also as the thickness $\delta_{f}$ of a film of water offering an equal thermal resistance. Based on measurements reported in previous analyses [14], a plausible value for the evaporation coefficient is approximately 0.1. Using this value, for water at atmospheric pressure the evaporative heat transfer coefficient is about $1 \mathrm{MW} \mathrm{m}^{-2} \mathrm{~K}^{-1}$. The associated thermal resistance is equal to that presented by a film of liquid water of thickness 1.0 micron.

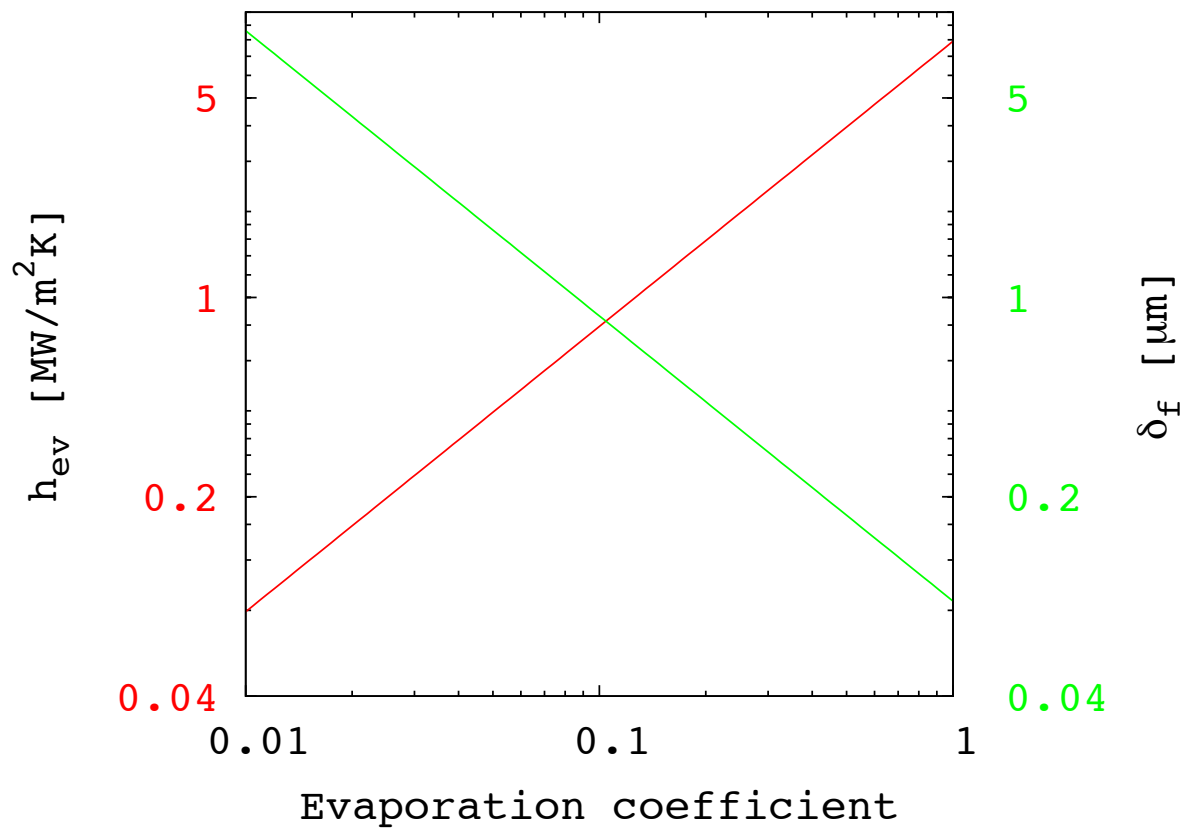

Figure 4

The evaporative heat transfer coefficient for water at atmospheric saturation conditions (left axis), and expressed as the thickness of water film of equivalent thermal resistance (right axis), as a function of the evaporation coefficient.

Another way to look at the interface thermal resistance is considering the interface superheat $S$ required to evaporate a given mass flux, Figure 5. 
For steam - water boiling at atmospheric pressure, a typical value of surface mass flux might be about $0.01 \mathrm{~kg} / \mathrm{m}^{2} \mathrm{~s}$ for the evaporation rate at the bubble curved surface, with micro-layer fluxes more than fifty times this, perhaps $0.5 \mathrm{~kg} / \mathrm{m}^{2} \mathrm{~s}$ [19]. With an evaporation coefficient of 0.1 , the interface needs to be superheated by a negligible $0.03 \mathrm{~K}$ in order to attain an evaporation rate representative of evaporation at bubble surface. This suggests that the interface resistance can be ignored when modelling evaporation at the bubble surface, which is the common approach. However, interface resistance causes a superheat of approximately $1.5 \mathrm{~K}$ to be required to attain fluxes typical of micro-layer evaporation.

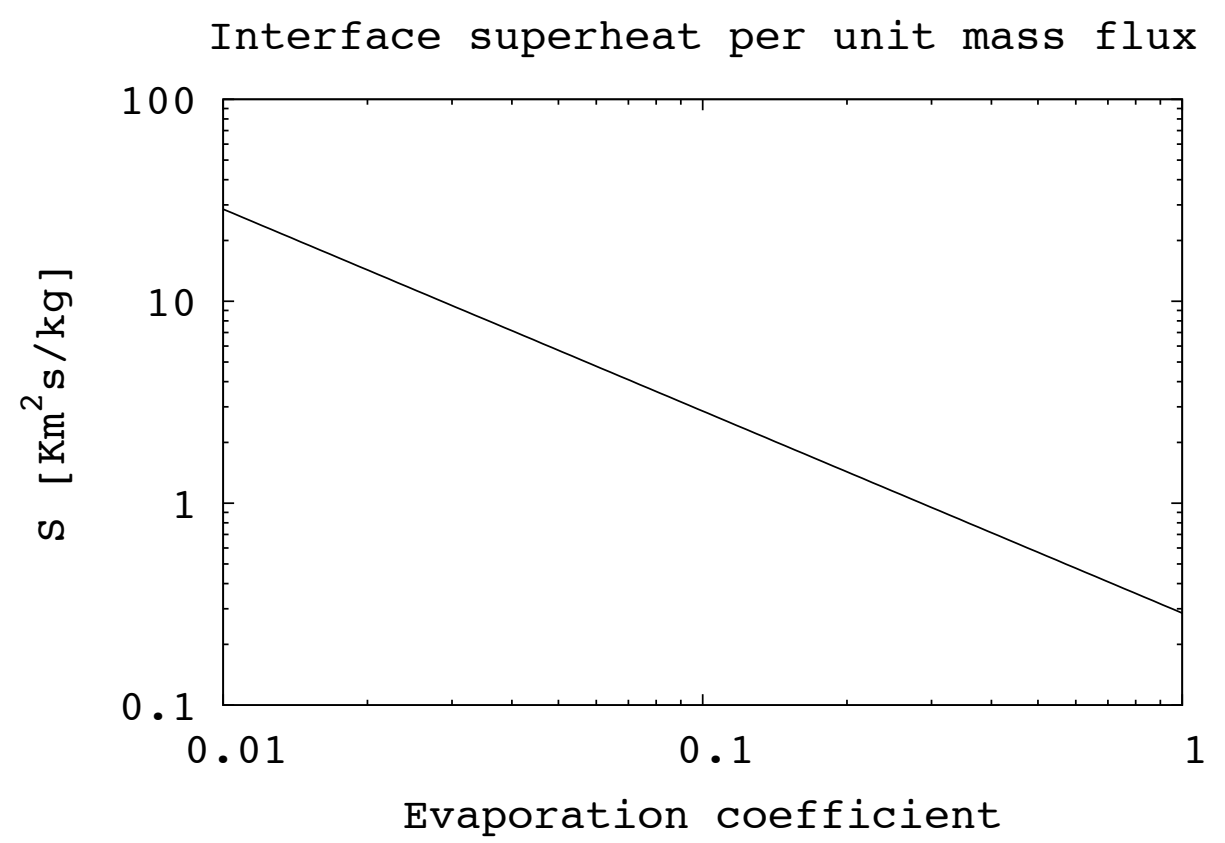

Figure 5

Interface superheat per unit mass flux, for water at atmospheric pressure as a function of the evaporation coefficient.

\subsection{Importance of the evaporative thermal resistance during bubble growth}

We now focus on the importance of the evaporative thermal resistance during bubble growth, and provide an order of magnitude argument in order to assess its importance as regards the two evaporation processes discussed in Section 0.

\subsubsection{Evaporation from the curved bubble surface}

For evaporation at the bubble surface, the thickness of the relaxation thermal layer increases with time, and is given by [20]:

$$
\delta_{T}(t)=\frac{R(t)}{\left(\frac{\rho_{l} C_{p_{l}} \Delta T}{\rho_{v} h_{f g}}\right)}=\frac{R(t)}{J a}
$$

where $R(t)$ is the bubble radius, $\rho_{l}$ is the liquid density, $c_{p l}$ the liquid specific heat capacity, and $\Delta T$ is the temperature difference between the liquid outside the thermal layer and the bubble surface. The denominator may be recognized as the Jakob number, Ja. 
The temporal variation of bubble radius can be obtained from the asymptotic solution for diffusion controlled bubble growth [20]:

$$
R(t)=J a \sqrt{\frac{12}{\pi} \alpha_{l} t}
$$

where $\alpha_{l}=k_{l} / \rho_{l} c_{p l}$ is the thermal diffusivity of the liquid and $k_{l}$ is the thermal conductivity of the liquid. This gives the time dependent relaxation thickness as:

$$
\delta_{T}(t)=\sqrt{\frac{12}{\pi} \alpha_{l} t}
$$

We then identify the ratio between evaporative thermal resistance and thermal resistance of the relaxation layer that opposes the flow of heat into the bubble as

$$
\Omega=\frac{\frac{1}{h_{e v}}}{\frac{\delta_{T}(t)}{k_{l}}}=\frac{\frac{1}{h_{e v}}}{\sqrt{\frac{12}{\pi \rho_{l} c_{p l} k_{l}} t}}
$$

This expression is plotted in Figure 6, where the symbol $\Omega$ denotes the ratio (evaporative thermal resistance)/(thermal resistance of the relaxation layer).

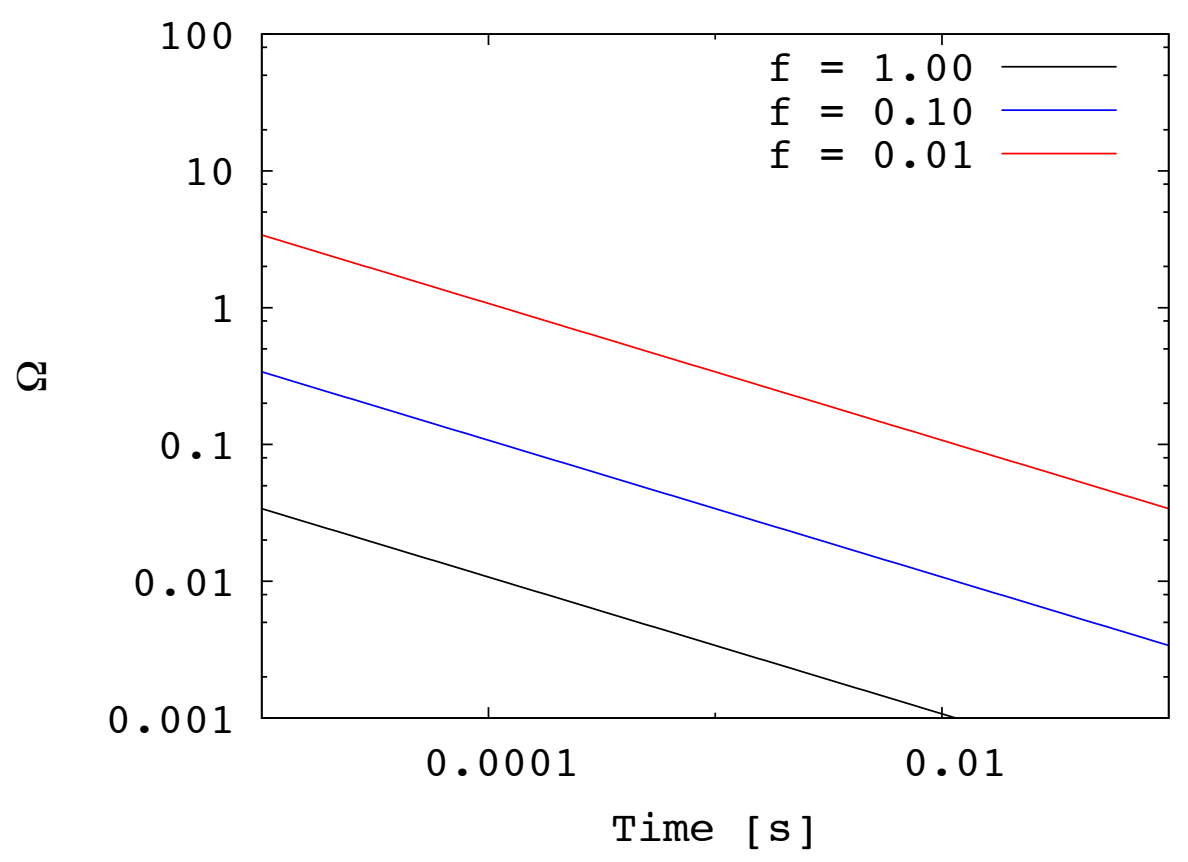

Figure 6

Evaporation at the bubble surface: The ratio (vertical axis) between evaporative thermal resistance and thermal resistance of the relaxation layer as it varies with time during bubble growth, for various evaporation coefficients.

Even for the smallest evaporation coefficient considered, the evaporative resistance declines rapidly to less than $10 \%$ of the total resistance. Typical bubble growth times for water at atmospheric pressure are of order hundredths of seconds, and the time for which the evaporative thermal resistance is the dominant resistance to the flow of heat into the bubble is 
very much smaller. This suggests that the effect of the evaporative resistance should be considered only during the very early stages of bubble growth. A similar conclusion was reached by Bornhorst et al. via a different approach [21].

\subsubsection{Evaporation from the micro-layer}

We now consider micro-layer heat transfer during the boiling of water at atmospheric pressure. Since the micro-layer thickness generally does not exceed five microns, the microlayer could offer resistance to heat transfer by conduction that is comparable to the evaporative resistance.

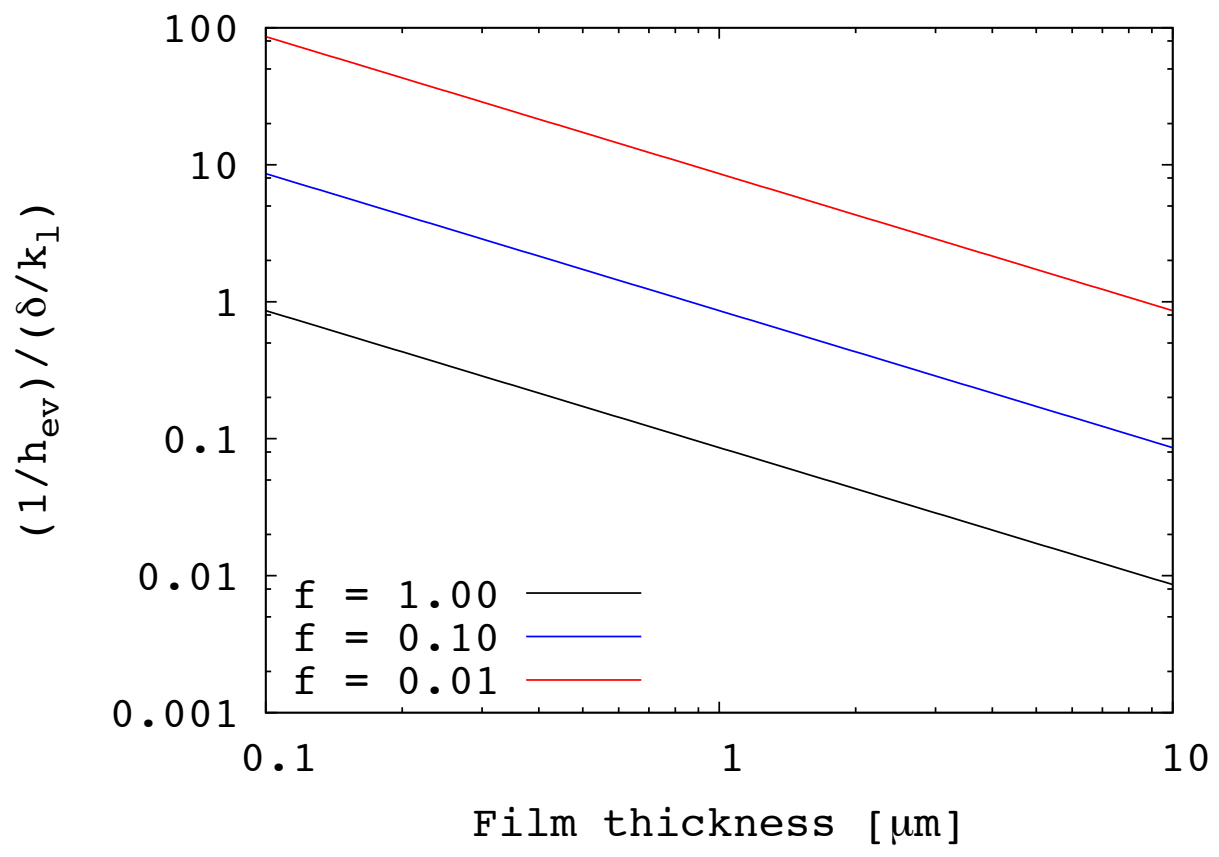

Figure 7

The ratio between evaporative thermal resistance and the thermal resistance of a thin film of water, for various evaporation coefficients.

The ratio between the evaporative thermal resistance and the micro-layer thermal resistance is plotted in Figure 7 as a function of film thickness, for a range of values of evaporation coefficient. This shows clearly that as the micro-layer thickness decreases, the evaporative resistance becomes increasingly important.

\section{MEASURED MICRO-LAYER EVAPORATION RATES; THE POOL BOILING TEST CASE OF JUNG AND KIM}

In what is perhaps one of the most comprehensive sets of measurements of bubble nucleation and growth, Jung and Kim [9] reported detailed measurements of the thickness and radius of the micro-layer as it varied during the bubble growth process. These provide an excellent basis for the investigation of the significance of the evaporative resistance in modifying evaporation rates.

\subsection{The pool boiling test case of Jung and Kim [9]}

The working fluid is water, boiled at atmospheric pressure using a $700 \mathrm{~nm}$ thick Indium-Tin Oxide (ITO) film heater deposited on top of a $10 \mathrm{~mm}$ thick calcium fluoride substrate. The 
ITO heater surface is $8 \mathrm{~mm} \times 15 \mathrm{~mm}$, with the boiling chamber a cylinder of base diameter $50 \mathrm{~mm}$. The average heat flux over the heater is $200 \mathrm{~kW} / \mathrm{m}^{2}$.

This set of measurements clearly demonstrated the existence of the micro-layer beneath a vapour bubble growing in water at atmospheric pressure. Jung and Kim were able to measure bubble shapes, micro-layer profiles and the temperature distribution on the underside of the ITO layer by means of, respectively, synchronized high speed imaging, laser interferometry and infrared thermometry; see Figure 8. Full details of the measurements are available in [19].

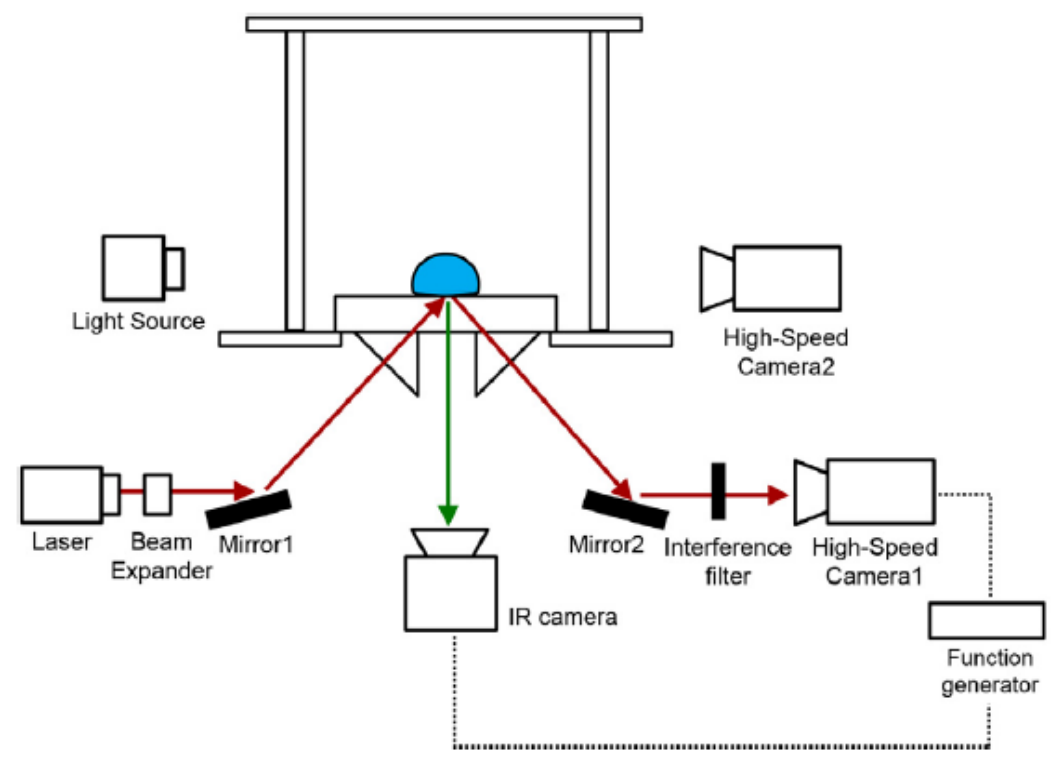

Figure 8

Schematic of Jung and Kim's experimental arrangements.

\subsection{Jung and Kim micro-layer measurements}

For present purposes the most important measurements are the series showing the development of the micro-layer. Results are presented of the radial variation of micro-layer evaporation rate, expressed as the rate of micro-layer thickness reduction, at a series of times. These measurements were obtained by two independent means:-

(i) Under the assumption that the liquid in the micro-layer is stagnant, optical interferometry allows the radial variation of micro-layer thickness to be measured directly at sequential times.

(ii) Measurement of the solid-liquid interface temperature through time allows the timedependent variation of the surface heat flux in the solid substrate to be determined by highly accurate finite element "post-processing" of the temperature field within the substrate. On the assumption that all of this surface heat flux goes into evaporating the micro-layer at the same location, the rate of thinning of the micro-layer can be determined.

(iii) A third 'derived' assessment of micro-layer thickness is also presented. Given the measurement of the solid surface temperature and the micro-layer thickness, and the assumption that the micro-layer upper surface temperature was equal to the saturation temperature, the heat flux through the micro-layer can be determined. From this the evaporation rate, and the rate of thinning of the micro-layer, can thereby be computed. 
We reproduce here (Figure 9) these measured quantities (from [19]), showing the micro-layer depletion rate assessed by the two direct measurements, and the third inferred method.

We will for brevity term these respectively:-

- Evaporation Heat Flux (i)

- Wall heat Flux (ii)

and the derived

- Conduction Heat Flux (iii).

The two independent measurements (i) and (ii) are in very good agreement with each other. For present purposes the other noteworthy feature of this graph is that the micro-layer depletion rate assessed by Method (iii) becomes inconsistent with the other two approaches at small radii, where the micro-layer is thin.

As discussed above, it is under these conditions that one would expect the neglect of surface evaporative resistance to begin to be significant. We will investigate whether or not inclusion of this phenomenon will restore consistency to the three approaches in the next section.

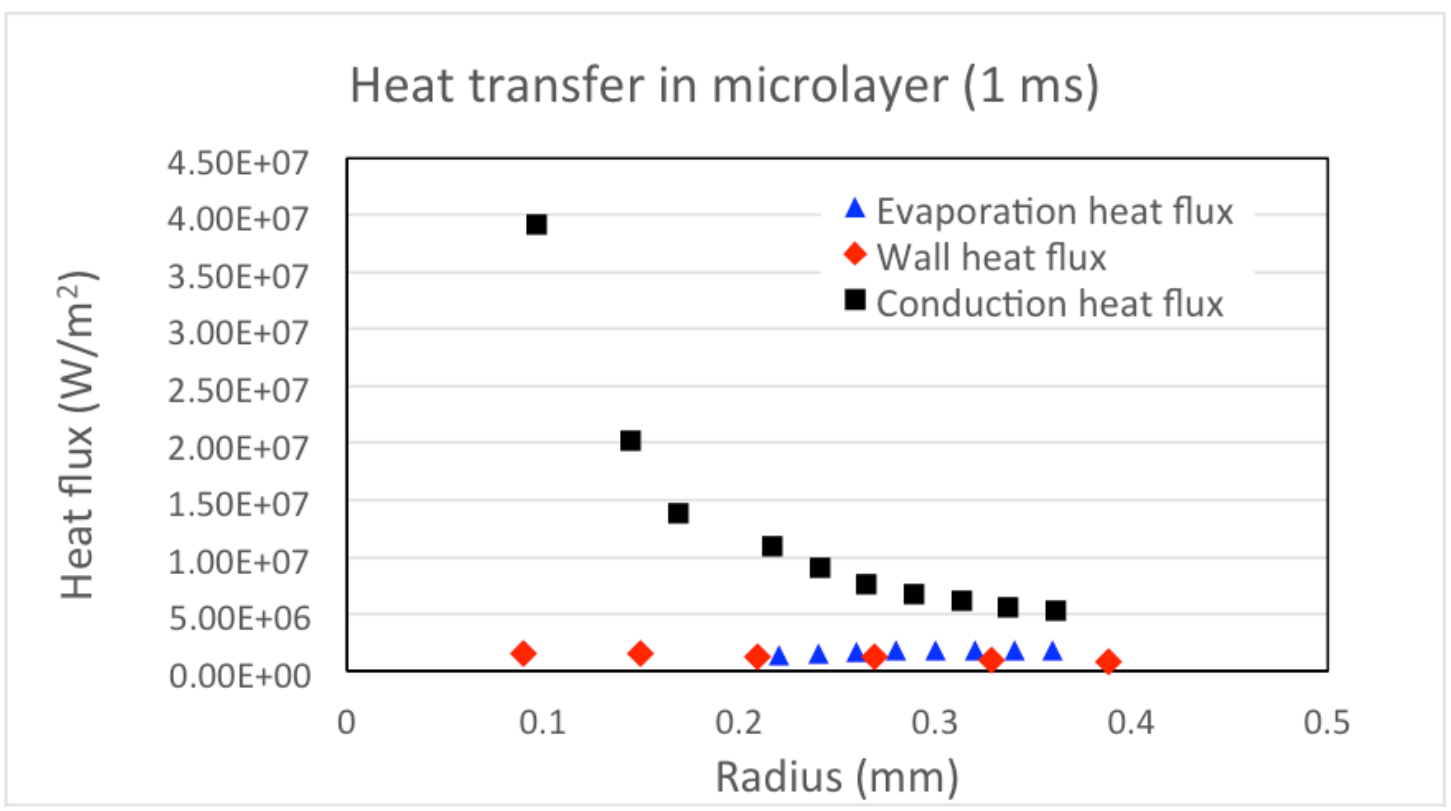



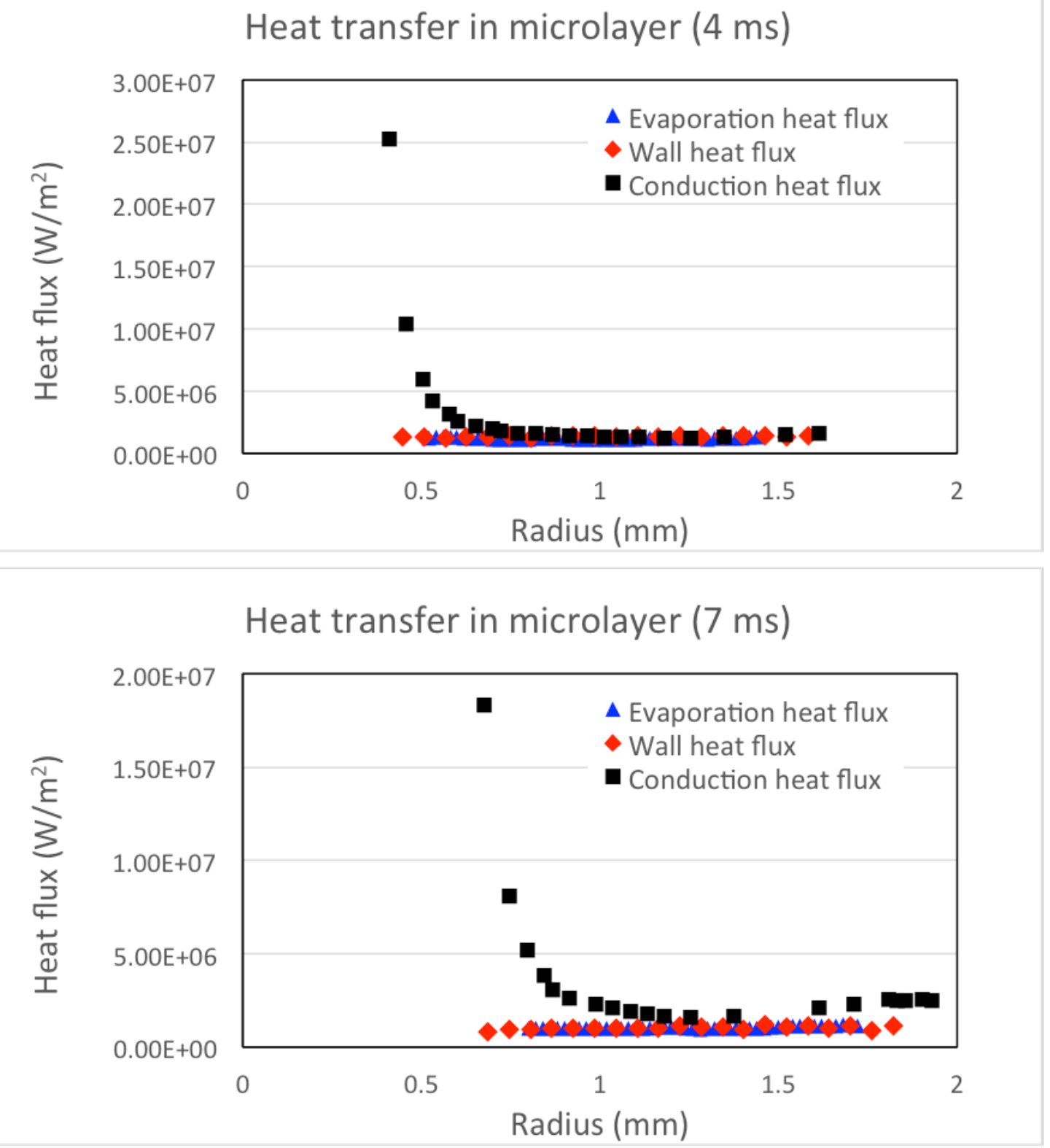

Figure 9

Reproduced from reference [19]. This shows the radial variation of micro-layer depletion rate reported by Jung and Kim, using the three different approaches discussed above. From top to bottom, the instantaneous micro-layer depletion profiles are shown at 1, 4 and $7 \mathrm{~ms}$ after bubble nucleation. 


\section{ANALYSIS OF THE JUNG AND KIM CASES}

4.1 Re-evaluation of the micro-layer heat flux radial and temporal variation incorporating evaporative thermal resistance

We can re-evaluate the heat flux via 'Method (iii)', but taking the evaporative thermal resistance into account, using the following expression:

$$
q(r, t)=\frac{T_{w}(r, t)-T_{S A T}}{\frac{\delta(r, t)}{k_{l}}+\frac{1}{h_{e v}}}
$$

where $T_{w}(r, t)$ is the local instantaneous value of the solid-liquid interface temperature and $\delta(r, t)$ is the micro-layer thickness at radius $r$ and time $t$. This is done for values of evaporative resistance and evaporation coefficient listed in Table 1, taken from the range of values believed to be credible, and results for the three times are shown in Figure 10.

\begin{tabular}{|l|c|l|}
\hline Case & Evaporative resistance $\left[\mathrm{m}^{2} \mathrm{~K} / \mathrm{W}\right]$ & Evaporation coefficient \\
\hline hev1 & $1.275 \times 10^{-7}$ & 1.00 \\
\hline hev2 & $1.275 \times 10^{-6}$ & 0.10 \\
\hline hev3 & $1.275 \times 10^{-5}$ & 0.01 \\
\hline
\end{tabular}

Table 1

Values of evaporative resistance considered.

It is seen that inclusion of the effect indeed can restore consistency between the 'derived' and the two independent 'measured' results, and that a value of $f$ lying between 0.1 and 0.01 seems appropriate. The very high value of heat flux predicted by Method (iii) close to the dry patch (where the micro-layer is becoming very thin) decreases significantly with progressively larger values of evaporative resistance. 

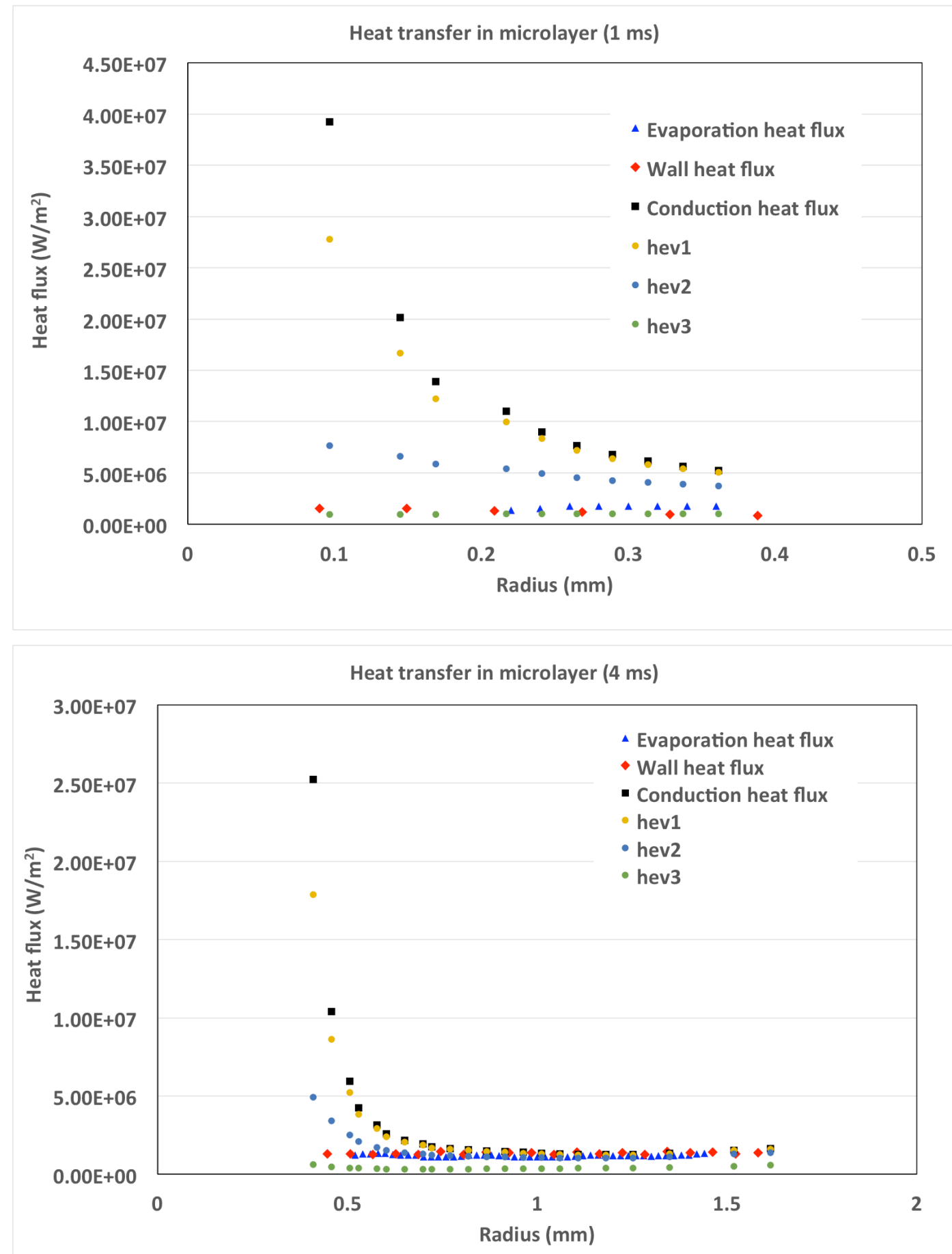


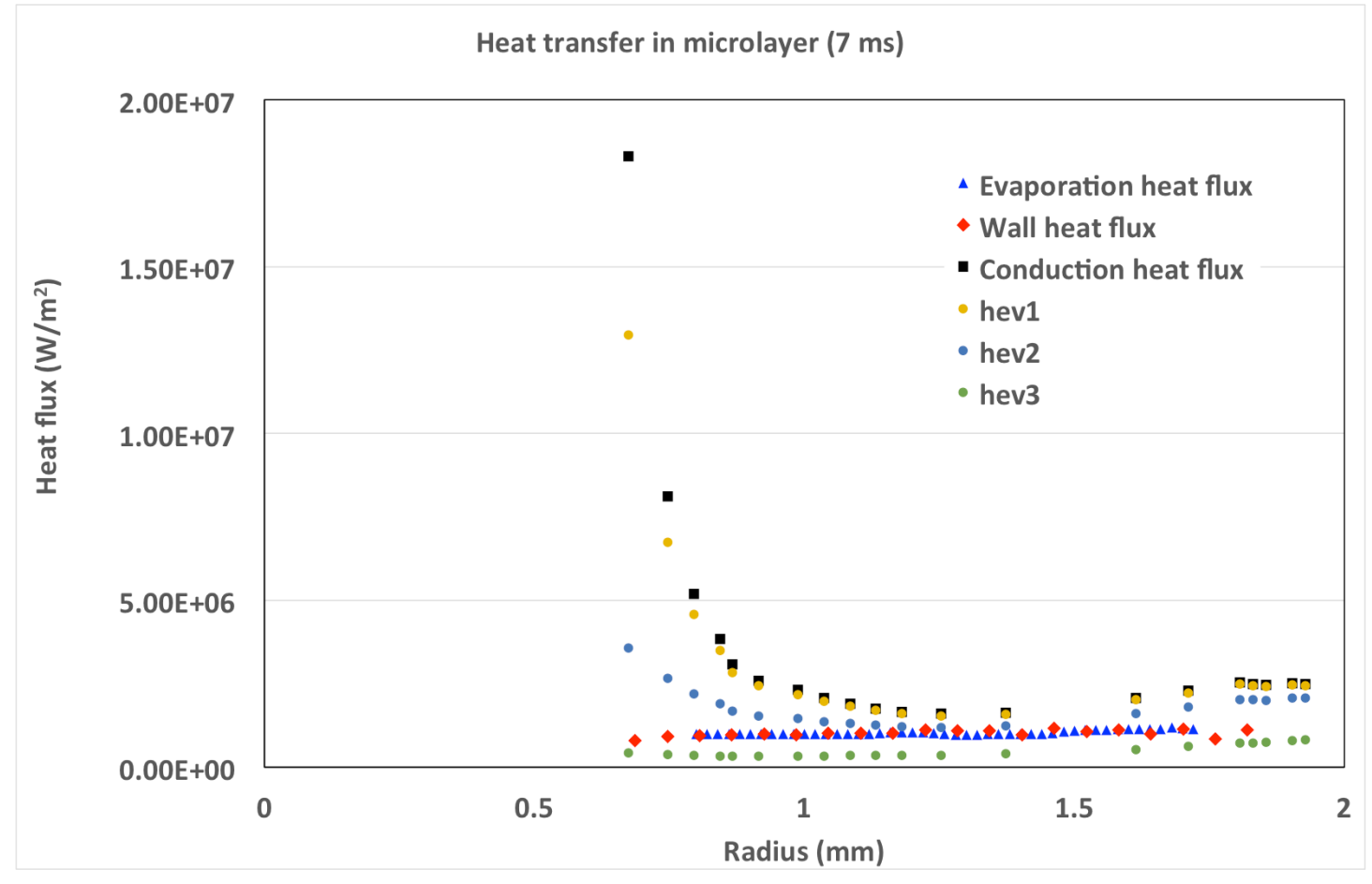

Figure 10

Influence of evaporative resistance on micro-layer depletion. 


\subsection{Inferring of evaporation coefficient value}

In the discussion above we employed a range of values of evaporation coefficient, and inferred by inspection a plausible value. In this section we will approach this more systematically, and compute directly the value required to generate a 'derived' heat flux consistent with the two measurements. (Note, of course, that as the two measurements are naturally not perfectly consistent with each other, the consistency of the derived approach has a similar degree of tolerance).

The approach adopted is:-

Knowing the wall temperature and the heat flux, the micro-layer upper surface temperature is computed:

$$
T_{I N T}(r, t)=T_{w}(r, t)-\frac{\delta(r, t) q(r, t)}{k_{l}}
$$

Using this temperature, the evaporative resistance is computed:

$$
\frac{1}{h_{e v}}=\frac{q}{T_{I N T}-T_{v}}
$$

The evaporation coefficient is inferred (using equation (5)), assuming the vapour at the saturation temperature at the system ambient pressure $p_{r e f}$ :

$$
f_{\text {inferred }}=\frac{q / h_{f g}}{\frac{p_{S A T}\left(T_{I N T}\right)}{\sqrt{2 \pi R T_{I N T}}}-\frac{p_{r e f}}{\sqrt{2 \pi R T_{S A T}\left(p_{r e f}\right)}}}
$$

where the saturation pressure at the interface temperature is computed according to the perfect gas law:

$$
p_{S A T}\left(T_{I N T}\right)=p_{r e f} \exp \left[\frac{h_{f g}}{R}\left(\frac{1}{T_{S A T}\left(p_{r e f}\right)}-\frac{1}{T_{I N T}}\right)\right]
$$

and $p_{\text {ref }}=101325 \mathrm{~Pa}$ (vapour assumed at system ambient pressure) and $T_{S A T}\left(p_{\text {ref }}\right)=373.15$ $\mathrm{K}$.

We have used this approach to compute the evaporative thermal resistance needed for consistency at each measurement location, at each time. Values are shown in Figure 11. Note that one would expect this assessment to become less well conditioned in regions where the micro-layer is thicker, and the evaporative thermal resistance is correspondingly a smaller fraction of the total thermal resistance. More weight should thus perhaps be given to values towards the inner radius. 


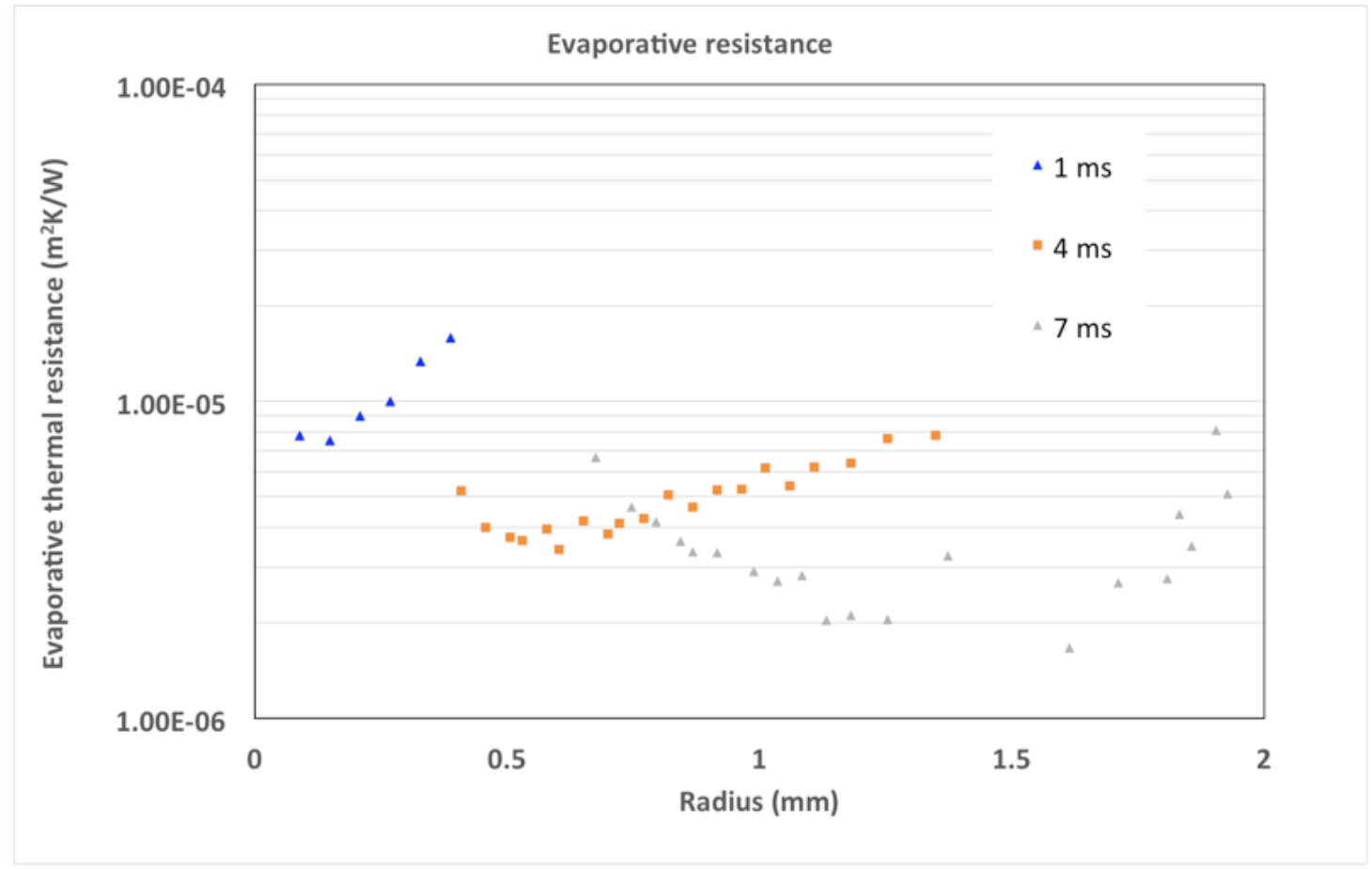

Figure 11

Value of the evaporative thermal resistance inferred from experimental data at 1, 4 and $7 \mathrm{~ms}$ after bubble nucleation.

In any event, it seems reasonable to infer a value of evaporative thermal resistance between 2 $\times 10^{-6}$ and $10 \times 10^{-6} \mathrm{~m}^{2} \mathrm{~K} / \mathrm{W}$. At small radii, where the micro-layer is thinner (and thus evaporative resistance more important), very similar values of evaporative resistance are obtained at each of the three times considered (respectively, 5, 7 and $8 \times 10^{-6} \mathrm{~m}^{2} \mathrm{~K} / \mathrm{W}$ ), an indication of the robustness of the present approach. 


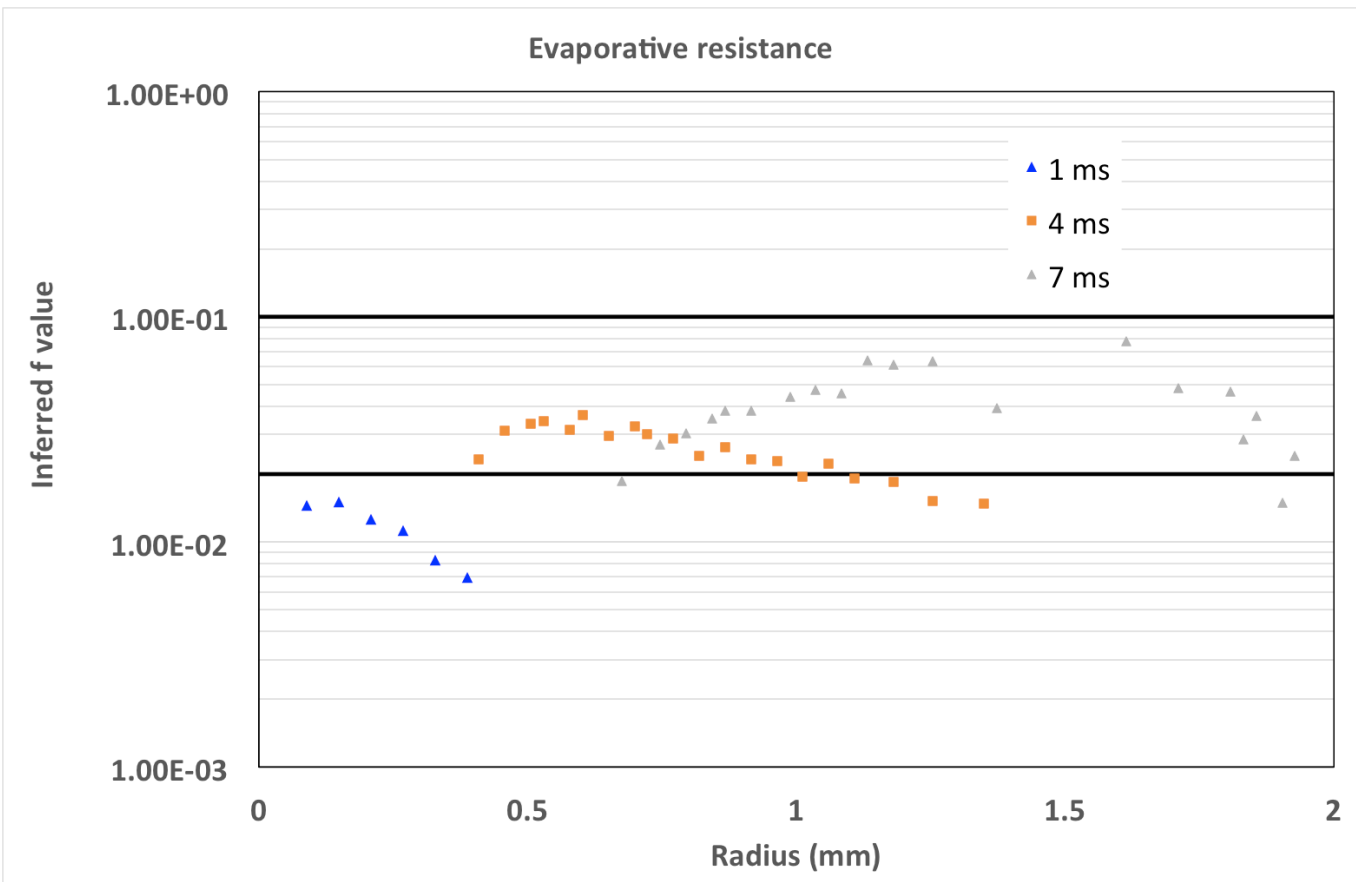

Figure 12

Evaporation coefficient inferred from experimental data at 1, 4 and $7 \mathrm{~ms}$ after bubble nucleation.

The approach can be repeated, to estimate a plausible range of values for the evaporation coefficient itself (Figure 12), giving corresponding approximately bounding values 0.02 and 0.1. These values are indicated on the figure (black lines). The evaporation coefficient inferred with measurements from to points close to the inner micro-layer radius is very similar at each of the three times considered, and close to 0.02 .

The degree of non-uniformity with respect to both location and time is quite significant, and seems to be greater than, for example, mere experimental uncertainty might cause to be seen.

As discussed above, the basic concept of "accommodation coefficient" by construction corresponds to the assumption that the evaporative process is able to be described by a "evaporative heat transfer coefficient", relating the heat flux linearly to the difference between the liquid surface temperature and the bulk vapour (saturation) temperature. One possible explanation would be if the evaporative process were not actually able to be so described, but rather displays a different dependence on this temperature difference. This is an interesting possibility, as it would cast doubt on the 'accommodation coefficient' approach, and it thus seems an area requiring further investigation.

Using bounding values for $f, 0.02$ and 0.1 , we now show in Figure 13 an indication of the ratio 'evaporative / conductive' in the resistance to the flow of heat that is presented by the microlayer. It is seen that even for the higher evaporation coefficient (the dots), the evaporative resistance would be everywhere a significant contributor to the total thermal resistance. For the lower value (solid lines), evaporative resistance dominates the conductive. This suggests strongly that in general the sub-grid micro-layer models [22] used in bubble growth modelling should be augmented to include this effect. 


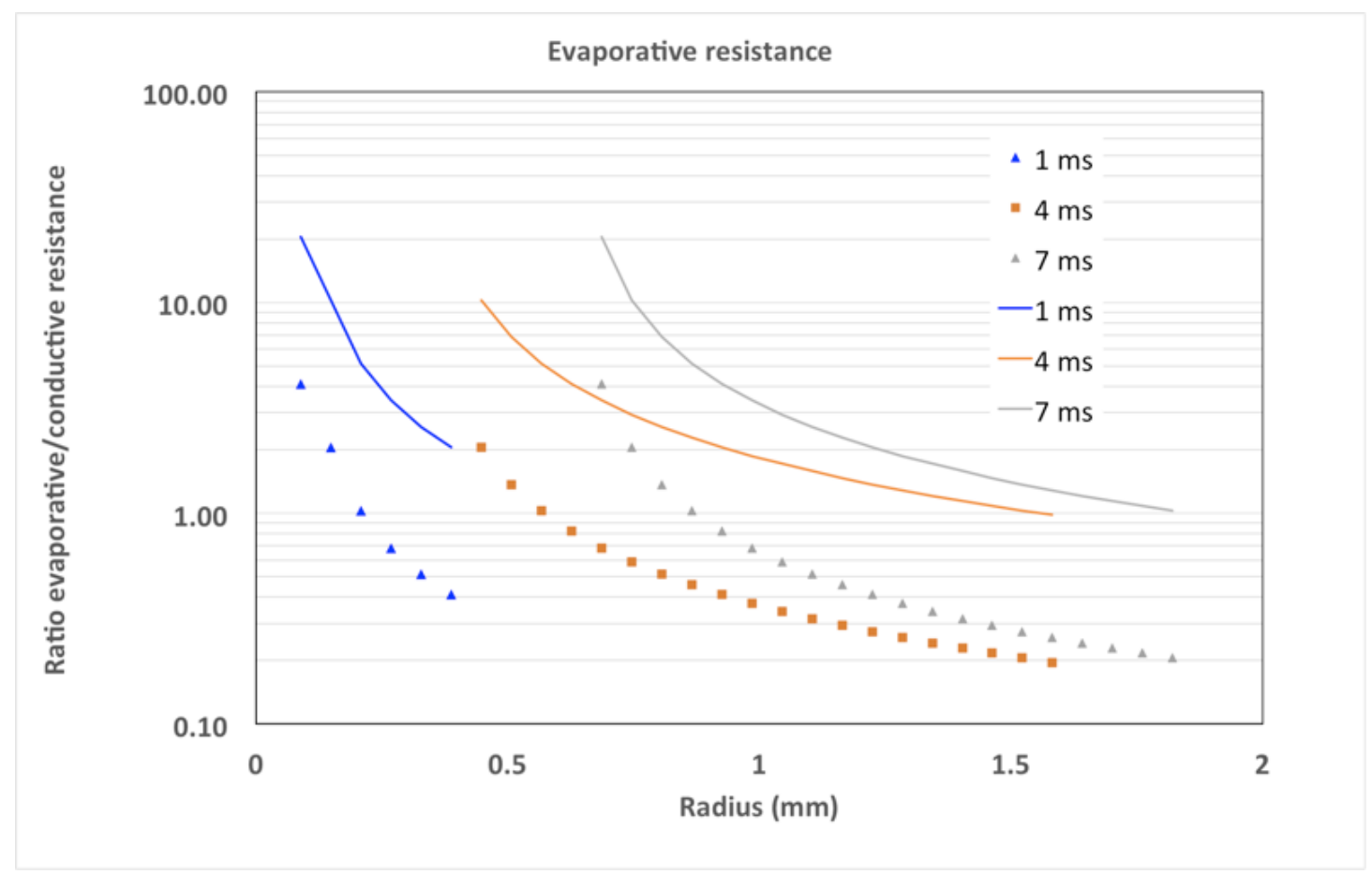

Figure 13

The micro-layer evaporative resistance relative to the conductive resistance at each of the three times, for the upper (points) and lower (solid lines) bounds to the evaporation coefficient

\section{CONCLUSIONS}

Micro-layer evaporative resistance is generally not included in microscopic modelling of vapour bubble growth. However, it has been shown in this work to be important in some circumstances, and in particular in the understanding of the evaporation of the micro-layer beneath the bubble. For many conditions, micro-layer evaporation is a significant contributor to the vapour forming the bubble. The flow of heat through this layer has been shown to be strongly affected by the evaporative 'heat transfer coefficient', associated with the molecular dynamics of the evaporation process at the vapour-liquid interface.

This has been demonstrated by analysis of the experiments of Kim, where the phenomenon is clearly shown to be able to restore consistency to the heat flux derived from two otherwise mutually self-consistent independent measurements.

Analysis of results at several times indicates that a quite constant value of evaporative heat transfer coefficient (and equivalently, of molecular evaporation coefficient) is able to achieve this consistency. Indeed, this result suggests that the experimental arrangements devised by Kim could actually provide a very promising tool to make deliberate, focused measurements of this rather difficult and elusive physical property.

\section{ACKNOWLEDGEMENTS}

It is a pleasure to acknowledge helpful discussion with Dr Vittorio Badalassi of Imperial College.

The financial support by Rolls Royce of one of us (Giovanni Giustini) is gratefully 
acknowledged.

\section{REFERENCES}

1. Dhir, V.K., Numerical Simulation of Pool Boiling: A Review. Journal of Heat Transfer, 2013. 135(6): p. 061502.

2. Kurul, N. and M.Z. Podowski, Multidimensional effects in forced convection subcooled boiling, in 9th International Heat Transfer Conference1990: Jerusalem, Israel. p. 21 - 25.

3. Rayleigh, L., VIII.On the pressure developed in a liquid during the collapse of a spherical cavity. Philosophical Magazine Series 6, 1917. 34(200): p. 94-98.

4. Scriven, L.E., On the dynamicsof phase growth. Chemical Engineering Science, 1958. 10(1/2).

5. $\quad$ Carey, V.P., Liquid-vapor phase-change phenomena. 1992: Hemisphere.

6. Cooper, M.G. and A.J.P. Lloyd, The microlayer in nucleate pool boiling. International Journal of heat and mass transfer, 1969. 12.

7. Guion, A., D. Langewisch, and J. Buongiorno, Dynamics of the liquid microlayer underneath a vapor bubble growing at a heated wall. Proceedingd of the Summer Heat Transfer Conference, July 14-19, 2013, Minnesapolis, MN, USA, 2013.

8. Utaka, Y., Y. Kashiwabara, and M. Ozaki, Microlayer structure in nucleate boiling of water and ethanol at atmospheric pressure. International Journal of Heat and Mass Transfer, 2013. 57(1): p. 222-230.

9. Jung, S. and H. Kim, An experimental method to simultaneously measure the dynamics and heat transfer associated with a single bubble during nucleate boiling on a horizontal surface. International Journal of Heat and Mass Transfer, 2014. 73: p. 365-375.

10. Koffman, L.D. and M.S. Plesset, Experimental Observations ofthe Microlayer in Vapor Bubble Growth on a Heated Solid. Journal of Heat Transfer, 1983. 105(3).

11. Hardt, S. and F. Wondra, Evaporation model for interfacial flows based on a continuumfield representation of the source terms. Journal of Computational Physics, 2008. 227(11): p. 5871-5895.

12. Jia, H.W., et al., A numerical investigation of nucleate boiling at a constant surface temperature. Applied Thermal Engineering, 2014.

13. Alexiades, V. and A.D. Solomon, Mathematical modelling of melting and freezing processes. 1993: Hemisphere.

14. Marek, R. and J. Straub, Analysis of the evaporation coeffi cient and the condensation coe fficient of water. International Journal of Heat and Mass Transfer, 2001. 44.

15. Schrage, R.W., A Theoretical Study of Interphase Mass Transfer 1953, New York: Columbia University Press.

16. Tanasawa, I., Condensation Heat Transfer, in Advances in Heat Transfer. 1991, Academic Press: San Diego, CA, USA.

17. Prueger, W., Die Verdampfungsgeschwindigkeit der Fluessigkeiten. Zeitschrift fr Physik, 1940. 115: p. 202 - 244.

18. Delaney, L.J., R.W. Houston, and L.C. Eagleton, The rate of vaporization of water and ice. Chemical Engineering Science, 1964. 19: p. 105 - 114.

19. Jung, S., S.H. Chung, and H. Kim, Experimental investigation of heat transfer mechanisms associated with the extended microlayer of a boiling bubble. 9th International Conference on Boiling and Condensation Heat Transfer, Boulder, Colorado, April 26-30, 2015. 
20. Plesset, M.S. and S.A. Zwick, The growth of vapor bubbles in superheated liquids. Journal of Applied Physics, 1954. 25(4).

21. Bornhorst, W.J. and G.N. Hatsopoulos, Bubble-Growth Calculation Without Neglect of Interfacial Discontinuities. Journal of Applied Mechanics, 1967.

22. Sato, Y. and B. Niceno, A depletable micro-layer model for nucleate pool boiling. Journal of Computational Physics, 2015. 300: p. 20-52. 